\title{
Neuroprotective and Therapeutic Role of Omega-3 Against Oxidative Stress and Neurotransmitter Disturbances in Rotenone-Induced Mice Model of Parkinson's Disease
}

\author{
Nagi Ali Ibrahim ${ }^{1, ~}$, Yasser Ashry Khadrawy ${ }^{2}$, Soliman Sayed Ibrahim ${ }^{1}$, Noura El-Sayed Ezzat ${ }^{1}$ \\ ${ }^{1}$ Zoology Department, Faculty of Science, Zagazig University, Zagazig, Egypt \\ ${ }^{2}$ Department of Medical Physiology, National Research Center, Dokki, Giza, Egypt \\ Email address: \\ Nagiibrahim_1050@yahoo.com (N. A. Ibrahim) \\ ${ }^{*}$ Corresponding author
}

\section{To cite this article:}

Nagi Ali Ibrahim, Yasser Ashry Khadrawy, Soliman Sayed Ibrahim, Noura El-Sayed Ezzat. Neuroprotective and Therapeutic Efficacy of Omega-3 Against Oxidative Stress and Neurotransmitter Disturbances in Brain of Rotenone-Induced Mice Model of Parkinson's Disease. Clinical Neurology and Neuroscience. Vol. 2, No. 1, 2018, pp. 12-22. doi: 10.11648/j.cnn.20180201.13

Received: November 16, 2017; Accepted: December 4, 2017; Published: February 7, 2018

\begin{abstract}
The present study aimed at evaluating the protective and therapeutic efficacy of omega-3 against motor impairment and brain biochemical disturbances in rotenone-induced mice model of Parkinson's disease (PD). Sixty animals were divided into six groups (10 each): mice of the $1^{\text {st }}$ group were used as controls, they were injected subcutaneously (sc) with the vehicle $(50 \mu \mathrm{l}$ dimethylsulfoxide (DEMSO) $+950 \mu \mathrm{l}$ sunflower oil $/ \mathrm{kg}$ body weight) every other day for 30 days; the $2^{\text {nd }}$ group, mice model of Parkinson's disease (PD), were injected (sc) with rotenone (3 mg/kg dissolved in vehicle every other day for 30 days). the $3^{\text {rd }}$ group, mice were given rotenone for 30 days followed by a stopping (recovery) period of other 30 days to validate the persistency of the PD model; the $4^{\text {th }}$ group (protection group), mice received orally Omega- 3 oil (300 $\mathrm{mg} / \mathrm{kg}$ ) daily an hour before every rotenone injection for 30 days; the $5^{\text {th }}$ and $6^{\text {th }}$ groups (therapeutic groups), mice were treated orally with Omega-3 oil daily for 7 and 15 days respectively after the induction of PD mice model. Data obtained revealed an impairment of the motor activity in mice of PD model as indicated from the decreased time of the forelimb hanging test. This was associated with a state of oxidative stress in the brain of PD model as indicated from the increase in lipid peroxidation (increased malondialdehyed, MDA, level) and nitric oxide (NO), and the decrease in reduced glutathione (GSH). A significant decrease in the levels of dopamine, norepinephrine, serotonin, AChE activity and a significant increase in TNF- $\alpha$ level was recorded in the PD model. The present findings show that both the protection by or oral treatment with omega- 3 for 15 days could ameliorate the rotenone- induced oxidative stress and inflammation in brain of PD mice model. In addition, omega-3 either as protection or treatment daily for 15 days was effective in restoring the decrease in dopamine and norepinephrine induced in the brain of PD mice model. In conclusion, the present study demonstrates that omega-3 supplementation potentially reverses the motor, and neurochemical alternations induced by rotenone in mice model of PD.
\end{abstract}

Keywords: Parkinson's Disease, Omega-3, Oxidative Stress, Neurotransmitters

\section{Introduction}

Parkinson's disease (PD) is the second most common neurodegenerative disorder after Alzheimer's disease, with a male -to -female ratio of about $3: 2$ in most studies $[1,2]$. Parkinson's disease is characterized by a loss of dopaminergic neurons in the substantianigra in the midbrain and also the presence of Lewy bodies, which are intracellular inclusions enriched in the protein $\alpha$-synuclein [3]. The most common motor symptoms of PD include bradykinesia, rest tremor, rigidity, and postural and gait impairment [4]. Various risk factors have been found for sporadic PD, including exposure to pesticides and other toxins, positive family history, and oophorectomy, but age remains the most important one documented so far $[1,5,6]$.

Parkinson's disease is a multifactorial disease characterized 
by self-perpetuating cascades involving a myriad of deleterious events at various stages including mitochondrial dysfunction, short-term and long-term oxidative and nitrosative stress, energy crisis, excitotoxicity, neuroinflammation and protein aggregation [7]. These events work together to promote cell death. Oxidative stress and mitochondrial dysfunction are responsible for the pathological features of $\mathrm{PD}$, such as neuronal death and apoptosis $[8,9]$.

On the other hand, PD is now considered a multisystem degenerative process, affecting not only the nigrostriatal dopaminergic system, responsible for the majority of the motor symptoms, but also other neurotransmitter systems, like cholinergic, serotonergic, and noradrenergic systems associated with several non motor symptoms such as sleep disturbances, fatigue, anxiety, depression, cognitive impairment, dementia, olfactory dysfunction, pain, sweating and constipation [10].

It is well known that the symptoms of PD worsen with time. The medications of PD provide the patients with a relief period from symptoms. These medications include levodopa [11], dopamine agonists [12], and catechol-Omethyl transferase (COMT) inhibitors that are added to levodopa treatment to overcome motor complications and to prolong the bioavailability of levodopa [13]. The anticholinergic drugs used in the treatment of PD are potentially helpful in delaying the need for levodopa treatment. In addition, they may allow for a reduction in the dose of levodopa required in more advanced cases, thus further extending the use of levodopa [14]. Although these medications could relief the symptoms of PD, many side effects have been reported including oxidative stress and the degeneration of residual dopamine neurons in patients with PD [15, 16, 17], confusion, hallucinations, depression, psychosis, dyskinesia, and mental changes [18]

It is clear that there is no exact cure for PD and many side effects have been reported due to the use of these medications. Therefore, there is a persistent need for new, safer and effective agents for the treatment of PD. Omega-3 polyunsaturated fatty acids (Omega-3 PUFAs), including eicosapentaenoic acid (EPA) and docosahexaenoic acid (DHA) are dietary fats with an array of health benefits [19]. DHA is a key component of all cell membranes and is found in abundance in the brain and retina [20]. EPA and DHA are the precursors of several metabolites that are potent lipid mediators, considered by many investigators to be beneficial in the prevention or treatment of several diseases [21]. These fatty acids are predominantly found in fish and fish oils [22].

Omega-3 PUFAs have a crucial role for optimal brain function by enabling fluidity in neuronal membranes and regulation of neurotransmitters [23]. DHA supplementation has been found to ameliorate memory and cognitive impairment in healthy older adults with age related cognitive decline without side effects [24]. It has been found that omega-3 supplementation may enhance resistance to free radical attack and reduce lipid peroxidation and may be an effective dietary supplement in the management of various diseases in which oxidant / antioxidant balance is disturbed as in aged brain tissue [25].
Bousquet et al. (2008) [26] found that omega-3 PUFAs had a partially protective effect against 1-methyl-4-phenyl-1, 2, 3, 6-tetrahydropyridine (MPTP)-induced reduction in dopamine production, storage, and/or release. It has been found that when rats are fed a diet deficient in omega-3 PUFAs, they displayed inadequate storage of newly synthesized dopamine [27]. Daily omega-3 PUFAs supplementation reversed the 5HT stress -induced effects but without impact on the physical state or behavior of the animals. This suggests that omega-3 PUFAs can improve resistance to stress [28].

Li et al. (2014)[29] found that marine derived omega-3 PUFAs supplementation had a significant lowering effect on the levels of C-reactive protein (CRP), interleukin 6 (IL-6) and tumor necrosis factor-alpha (TNF- $\alpha$ ) particularly in non obese subjects.

Although several approved drugs may alleviate PD symptoms, long term use of these drugs is often associated with aggravating side effects, and none of these drugs can slow down, prevent or even reverse the progress of PD. Therefore, the aim of the present study is to evaluate the protective and therapeutic effect of omega-3 against mice model of PD induced by rotenone. This is carried out by investigating the effect of omega-3 on the motor and neurochemical changes induced in the brain of mice PD model.

\section{Materials and Methods}

\subsection{Animals}

Forty eight Swiss male albino mice (weighing 30-40 g.) were used in the present study. The animals were obtained from Animal House Colony of National Research Centre, Giza, Egypt. On arrival, animals were housed ten per cage in stainless steel cages with ad libitum access to standard laboratory diet and tap water in a temperature-controlled (20$25^{\circ} \mathrm{C}$ ) and artificially illuminated (12 hrs. dark / light cycle) room free of any chemical contamination. All animals received human care in compliance with the guidelines of the Animals Care and Use Committee of the National Research Centre, Egypt, on July 2009 with registration number 09/093.

\subsection{Drugs and Chemicals}

Rotenone was purchased from Sigma Chemical Co. (St. Louis MO, USA). It was dissolved first in dimethylsulfoxide $(1.5 \mathrm{mg} / 50 \mu \mathrm{l})$ and then the volume was completed with sunflower oil to have a final concentration of $1.5 \mathrm{mg} / \mathrm{ml}$. Omega-3 oil was purchased from Arab Co. for Gelatin and Pharmaceutical Products, Egypt.

\subsection{Experimental Design}

Animals were divided into 6 groups, mice of the $1^{\text {st }}$ group were used as control, they received orally the vehicle $(50 \mu 1$ dimethylsulfoxide (DEMSO) $+950 \mu \mathrm{l}$ sunflower oil/kg body weight) every other day for 30 days; the $2^{\text {nd }}$ group, mice model of Parkinson's disease (PD), were treated with subcutaneous (sc) injection of rotenone $(3 \mathrm{mg} / \mathrm{kg}$ body weight. dissolved in dimethylsulfoxide + sunflower oil every other day for 30 days); 
the $3^{\text {rd }}$ group, mice were injected s c with rotenone for 30 days followed by a recovery period for other 30 days to investigate whether the mice model of PD could recover to normal or not after stopping rotenone injection; the $4^{\text {th }}$ group (protection group) mice received orally Omega-3 oil daily (300 mg/kg) an hour before every rotenone injection for 30 days; the $5^{\text {th }}$ and $6^{\text {th }}$ groups (therapeutic groups), mice were treated orally with Omega-3 oil daily for 7 and 15 days respectively after the induction of mice model of PD.

\subsection{Motor Activity (Hanging Wire Test)}

This test was used as a measure of muscular strength and motor neuron integrity. Mice used their forelimbs to suspend their body weight on a wire stretched between two posts 60 $\mathrm{cm}$ and $20 \mathrm{~cm}$ above a foam pillow. The time (in seconds) until the rat fell was recorded [30].

\subsection{Preparation of Brain Samples}

Following motor testing, animals were sacrificed by sudden decapitation after being fasted for 12 hours. The brain of each animal was quickly disected out and rapidly transferred to an ice-cold Petri dish. Each brain was divided into two right and left halves. The right half was used to measure oxidative stress parameters [Malondialdehyde (MDA), Nitric oxide (NO), reduced glutathione (GSH)], acetylcholineesterase (AchE) activity and tumor necrosis factor-alpha (TNF- $\alpha$ ) level. The left half was used for the analysis of other neurotransmitter including dopamin (DA), norepinephrine (NE) and serotonin (5-hydroxytryptamine; 5-HT). Each brain area was weighed and frozen at $-80^{\circ} \mathrm{C}$ until analyzed.

The right half of each mouse brain was homogenized in phosphate buffer ( $\mathrm{pH} 7.4)$. This homogenate was centrifuged at $5000 \mathrm{rpm}$ and $4^{\circ} \mathrm{C}$ for 10 minutes; the supernatant was stored at $-80^{\circ} \mathrm{C}$ until analysis. This supernatant was used for the determination of oxidative stress parameters, AchE activity and TNF- $\alpha$ level.

\subsection{Neurochemical Analysis}

\subsubsection{Determination of Lipid Peroxidation}

MDA, a measure of lipid peroxidation, was estimated according to the method of Ruiz-Larrea et al. (1994) [31]. Malondialdehyde was determined by measuring thiobarbituric acid reactive species (TBARS). One molecule of MDA reacts with two molecules of thiobarbituric acid in acidic medium at a temperature of $95^{\circ} \mathrm{C}$ for 20 minutes to form TBARS. The absorbance of the resultant pink product was measured at $532 \mathrm{~nm}$.

\subsubsection{Determination of $\mathrm{NO}$}

NO was determined colorimetrically in the brain tissue according to the method described by Montgomery and Dymock (1961) [32]. This is based on the measurement of endogenous nitrite concentration as an indicator of $\mathrm{NO}$ production. It depends on the addition of Griess reagent which converts nitrite into a deep purple azo compound whose absorbance is read at $540 \mathrm{~nm}$.

\subsubsection{Determination of GSH}

Ellman's method [33] was used to measure GSH level. The procedure is based on the reduction of Ellman's reagent by $-\mathrm{SH}$ groups of GSH to form 2-nitro-s-mercaptobenzoic acid whose intense yellow color is measured spectrophotometrically at 412 $\mathrm{nm}$.

\subsubsection{Determination of $T N F-\alpha$}

Estimation of brain TNF- $\alpha$ level expressed in $\mathrm{pg} / \mathrm{g}$ tissue was carried out using rat TNF- $\alpha$ ELISA Kit supplied by Koma Biotech INC, Seoul (Korea).

\subsubsection{Neurotransmitter Analysis}

The procedure used for the determination of AchE activity in the midbrain and striatum was a modification of the method of Ellman et al. (1961) [34] as described by Gorun et al. (1978) [35]. The principle of the method depends on the hydrolysis of acetylthiocholine iodide by acetycholinesterase to produce thiocholine. Thiocholine is allowed to react with the -SH reagent 5,5'-dithiobis-(2-nitrobenzoic acid) (DTNB), which is reduced to thionitrobenzoic acid, a yellow colored anion whose absorption is read spectrophotometrically at $412 \mathrm{~nm}$.

The left half of each mouse was homogenized in an icecold solution of acidified n-butanol. The homogenates were centrifuged at 2000 r. p. m. for 5 minutes. These supernatants were used for the estimation of DA, NE and 5-HT according to the fluorometric method described by Ciarlone (1978) [36]. The fluorescence was measured using spectrofluorometer (model Jasco-FP-6500, Japan) with a source of xenon arc lamp 150W (excitation slit band width of excitation monochromator: $5 \mathrm{~nm}$., emission slit band width of emission monochromator: $5 \mathrm{~nm}$.).

\subsection{Statistical Analysis}

The data presented in the study were statistically evaluated as a mean value for each group and its corresponding standard error. Statistical significance between the groups under investigation was tested by one-way analysis of variance (ANOVA) using Statistical Package for Social Sciences (SPSS) program, version 14 followed by post hoc test using Duncan to compare significance between groups. Difference was considered significant at P-value $<0.05$.

\section{Results}

\subsection{Motor Activity}

Data demonstrated in figure (1) revealed that rotenone injection ( $3 \mathrm{mg} / \mathrm{kg}$ every other day) for 30 days resulted in a significant decrease in the hanging time of mice below the control value. The daily oral protection with omega-3 (300 $\mathrm{mg} / \mathrm{kg}$ ) against rotenone induce model of PD restored the hanging time of mice to control -like value. Moreover, when the mice model of PD were treated daily with omega-3 for 7 and 15 days, the decrease in forelimb hanging time induced by rotenone returned to non-significant change as compared to control value. 


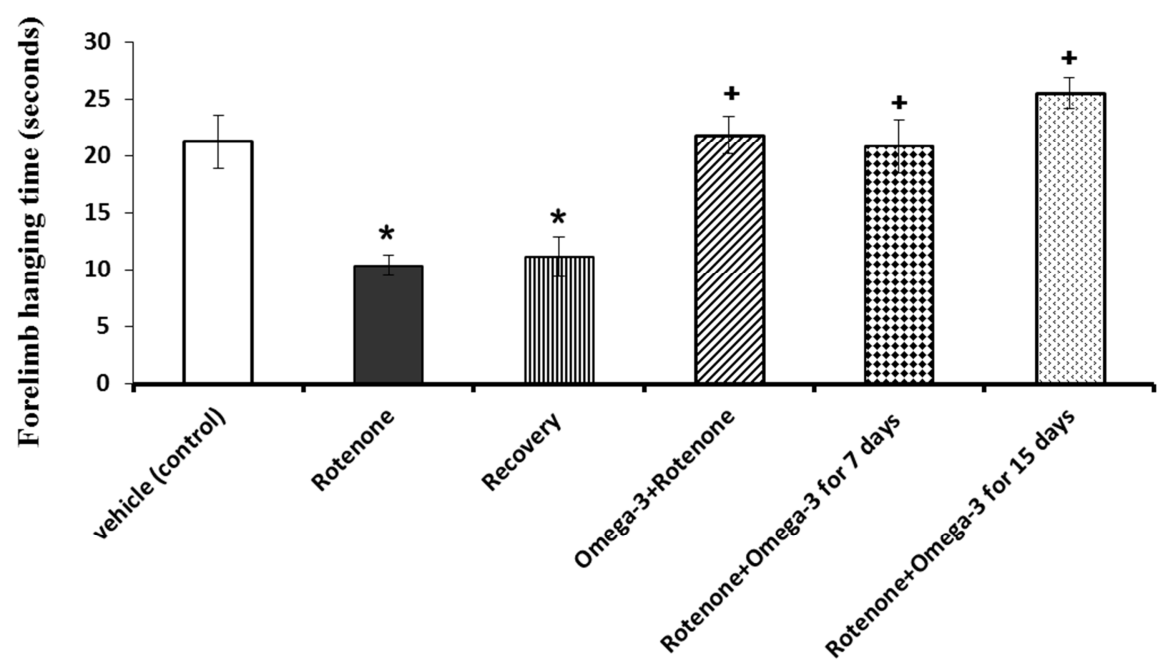

Figure 1. Effects of omega-3 protection or treatment on the motor activity (fore limp hanging time) in mice of rotenone induced PD model as compared to the vehicle (control) $) * P<0.05$ and rotenone ${ }^{+} P<0.05$ groups respectively.

\subsection{Neurochemical Results}

\subsubsection{Oxidative Stress Parameters}

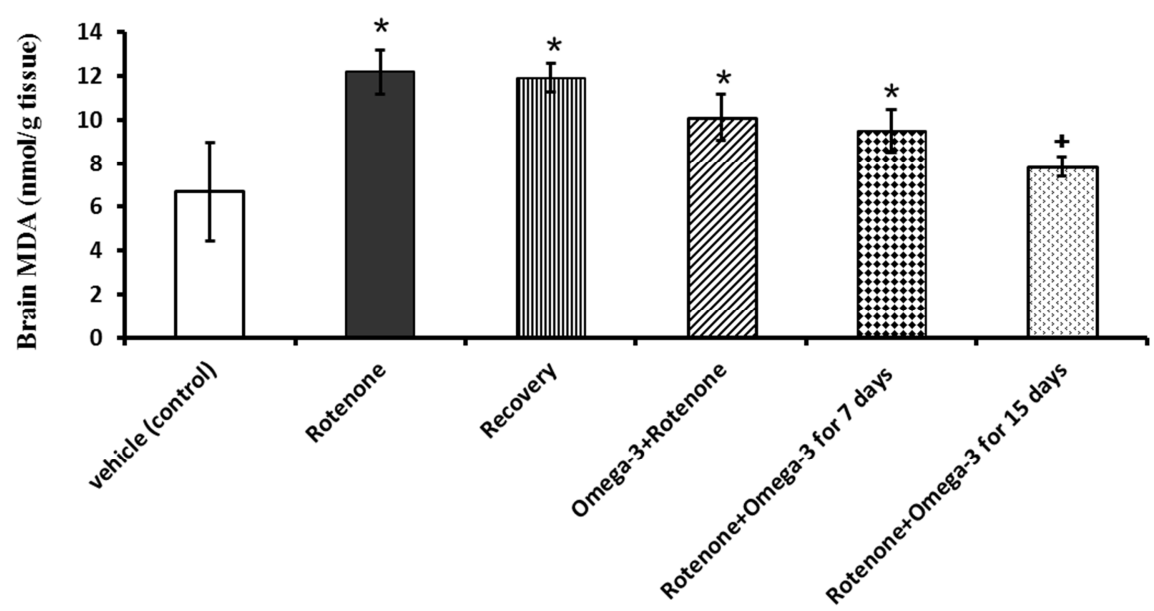

Figure 2. Effects of omega-3 protection or treatment on the level of MDA in mice brain of rotenone-induced PD model as compared to the vehicle (control)* $P<0.05$ and rotenone ${ }^{+} P<0.05$ groups respectively.

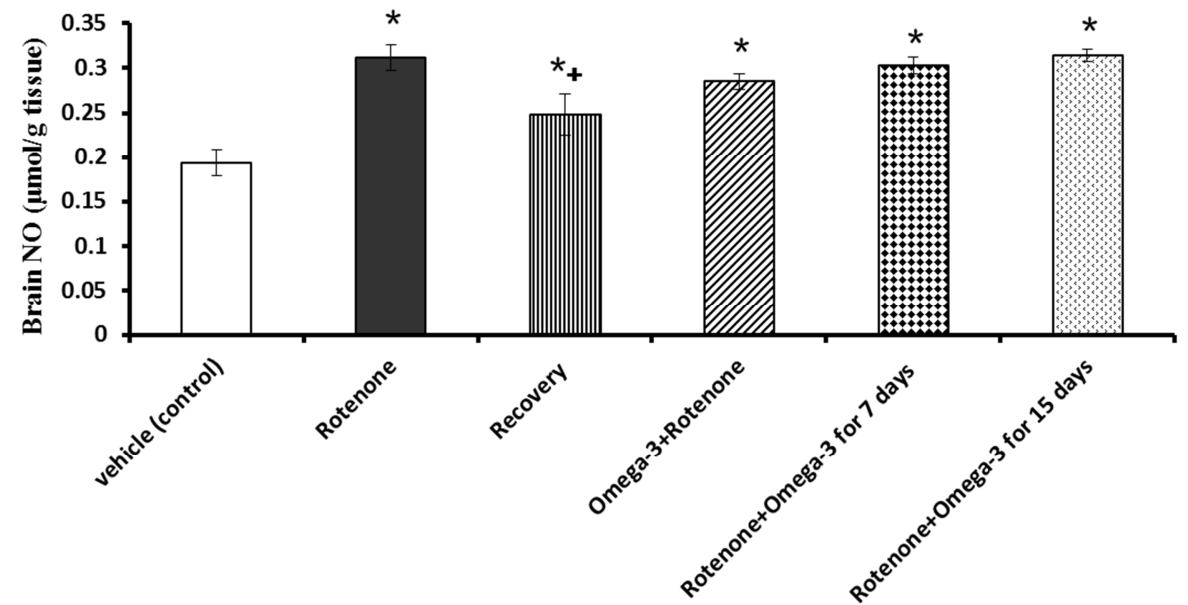

Figure 3. Effects of omega-3 protection or treatment on the level of NO in mice brain of rotenone-induced PD model as compared to the vehicle (control)* $P<$ 0.05 and rotenone ${ }^{+} P<0.05$ groups respectively. 


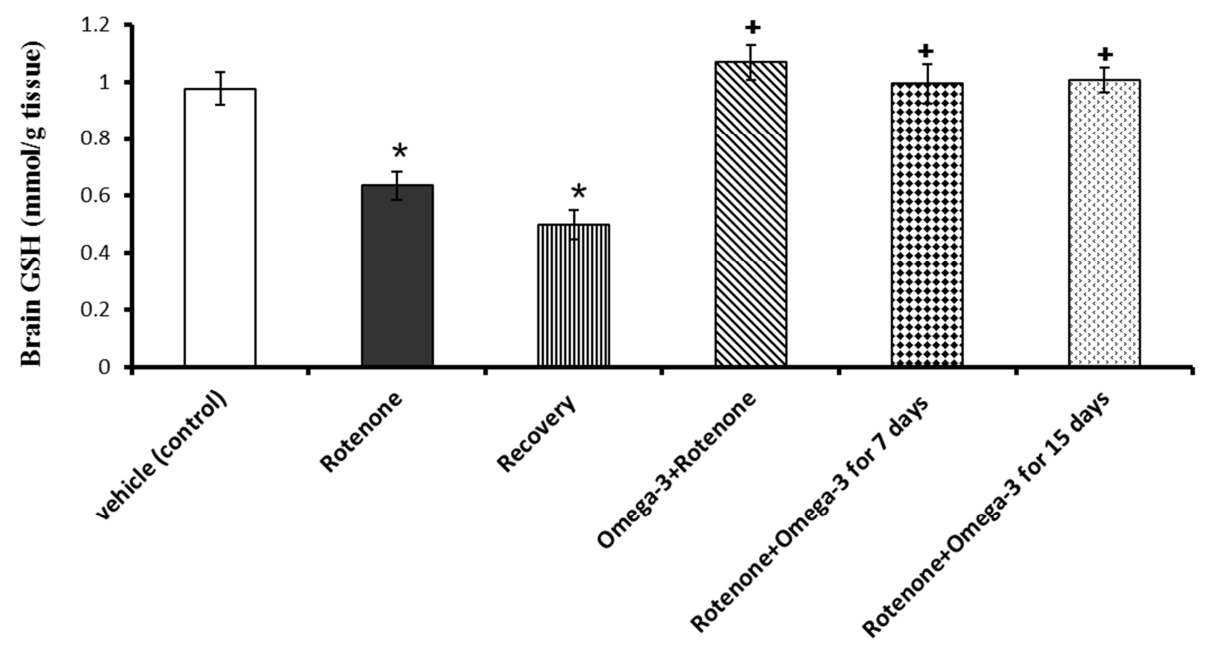

Figure 4. Effects of omega-3 protection or treatment on the level of GSH in mice brain of rotenone induced PD model as compared to the vehicle (control) * $P<0.05$ and rotenone ${ }^{+} P<0.05$ groups respectively.

Data illustrated in figures (2 and 3) show that rotenoneinduced mice model of PD was accompanied by significant increases in brain levels of lipid peroxidation (MDA) by $82.29 \%$ and NO by $60.82 \%$ respectively and a significant decrease in the level of GSH by $35.30 \%$ Figure (4) as all compared to their levels in control mice. The protection and daily treatment with Omega- 3 for 7 days failed to restore the increased levels of lipid peroxidation and nitric oxide induced in the brain of PD mice model, however, GSH value showed non significant change. Daily treatment of PD mice model with omega-3, for 15 days restored brain levels of both MDA and GSH toward the control values, whereas, NO showed elevated level (figures 2, 3 and 4) respectively.

Data demonstrated in figure (5) showed a significant increase in TNF- $\alpha$ by $33.6 \%$ in the brain of mice model of PD as compared to the control value. This increase was reduced to control like value when the mice model of PD were protected or treated daily for 15 days with omega-3.

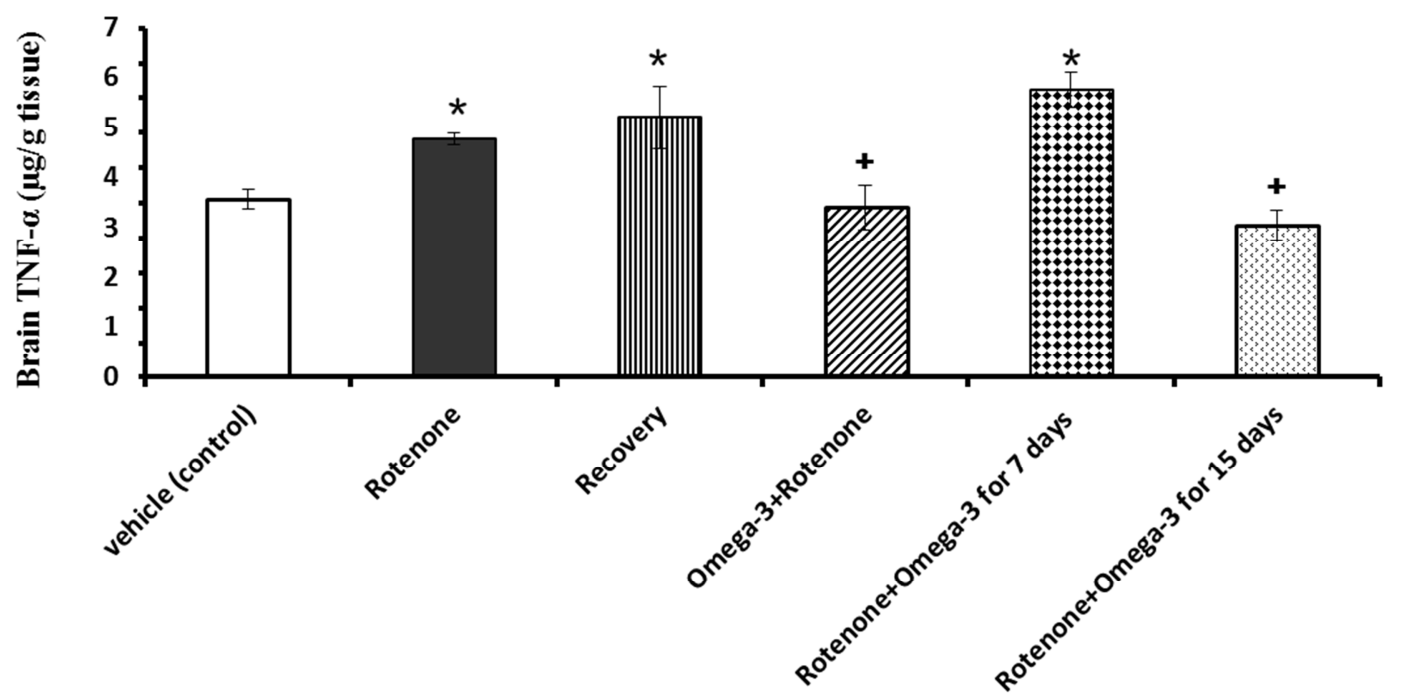

Figure 5. Effects of omega-3 protection or treatment on the level of TNF- $\alpha$ in mice brain of rotenone induced PD model as compared to the vehicle (control) * $P<0.05$ and rotenone ${ }^{+} P<0.05$ groups respectively.

\subsubsection{Neurotransmitters}

Figure (6) shows a significant decrease jn brain AchE activity by $-21.28 \%$ below the control value. Both the protection and treatment with omega- 3 for 7 days reversed the decrease in the AchE activity to a significant increase. However, treatment of mice model of PD with omega-3 was extended to 15 days AchE activity was restored to control like value.

In mice model of PD significant decreases in the brain levels of DA (figure 7), NE (figure 8) and 5-HT (Figure 9) were observed recording $-14.68 \%,-15.49 \%$ and $-58.71 \%$, respectively, as compared to control values. The protection with omega-3 prevented the decrease in dopamine and norepinephrine induced in mice model of PD but failed to restore the decrease in serotonin. Similarly the daily treatment of mice model of PD with omega-3 for 7 and 15 days restored the brain levels of DA and NE to non significantly changed as compared to control animals. However, omega-3 treatment was unable to prevent the decrease in 5-HT induced in mice model of PD. 


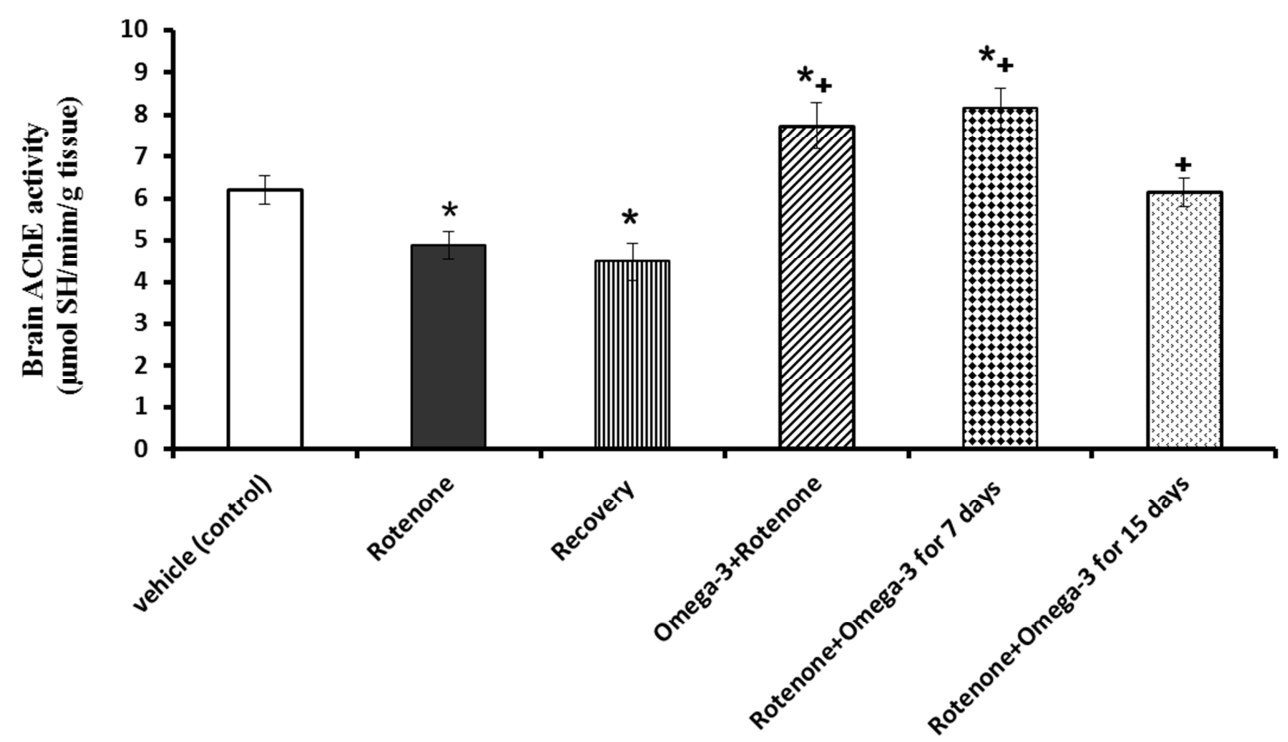

Figure 6. Effects of omega-3 protection or treatment on the level of AchE activity in mice brain of rotenone induced PD model as compared to the vehicle (control) $* P<0.05$ and rotenone ${ }^{+} P<0.05$ groups respectively.

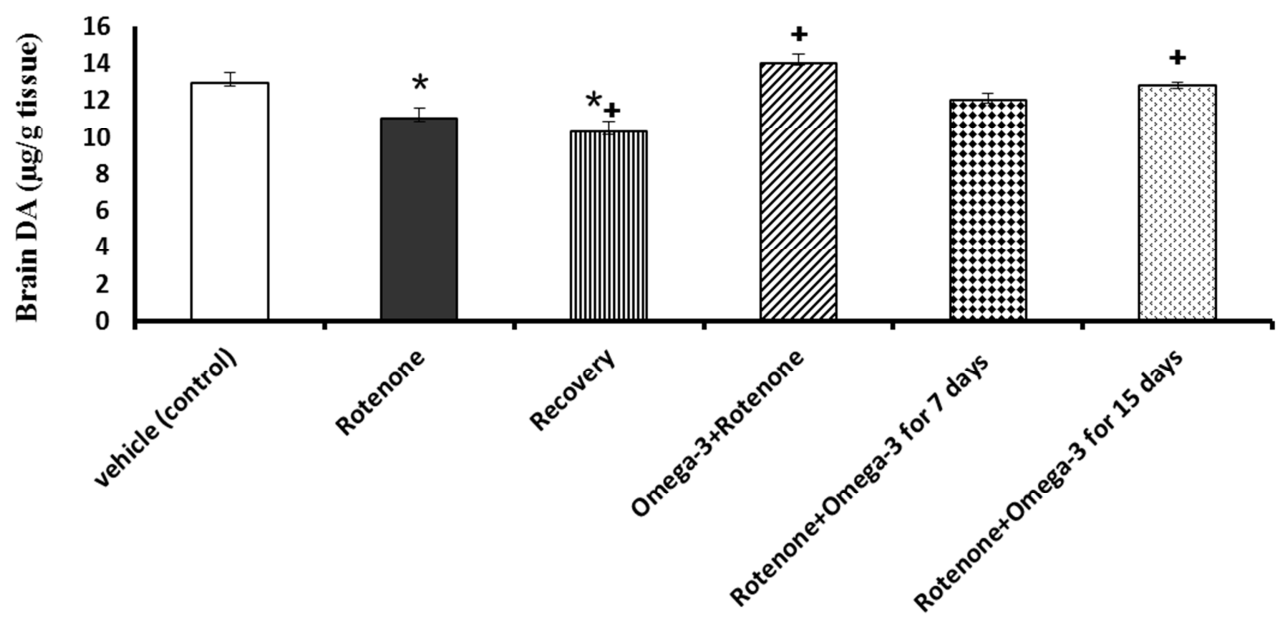

Figure 7. Effects of omega-3 protection or treatment on the level of DA in mice brain of rotenone induced PD model as compared to the vehicle (control). * $P<0.05$ and rotenone ${ }^{+} P<0.05$ groups respectively.

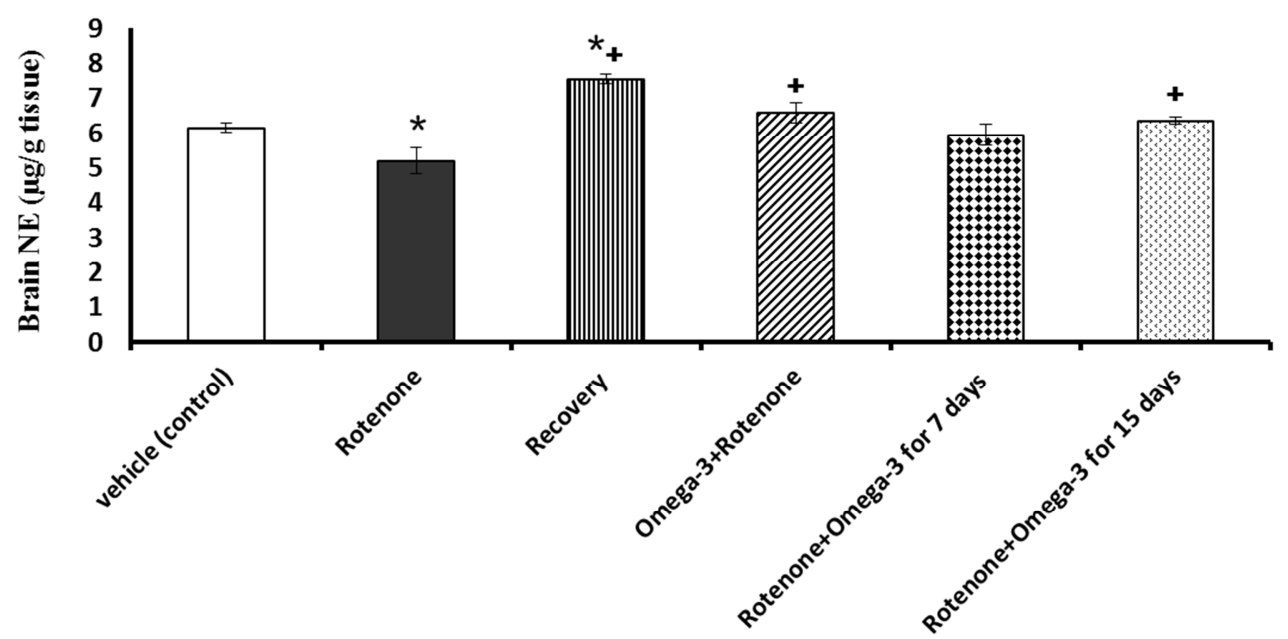

Figure 8. Effects of omega-3 protection or treatment on the level of NE in mice brain of rotenone-induced PD model as compared to the vehicle (control) * $P<$ 0.05 and rotenone ${ }^{+} P<0.05$ groups respectively. 


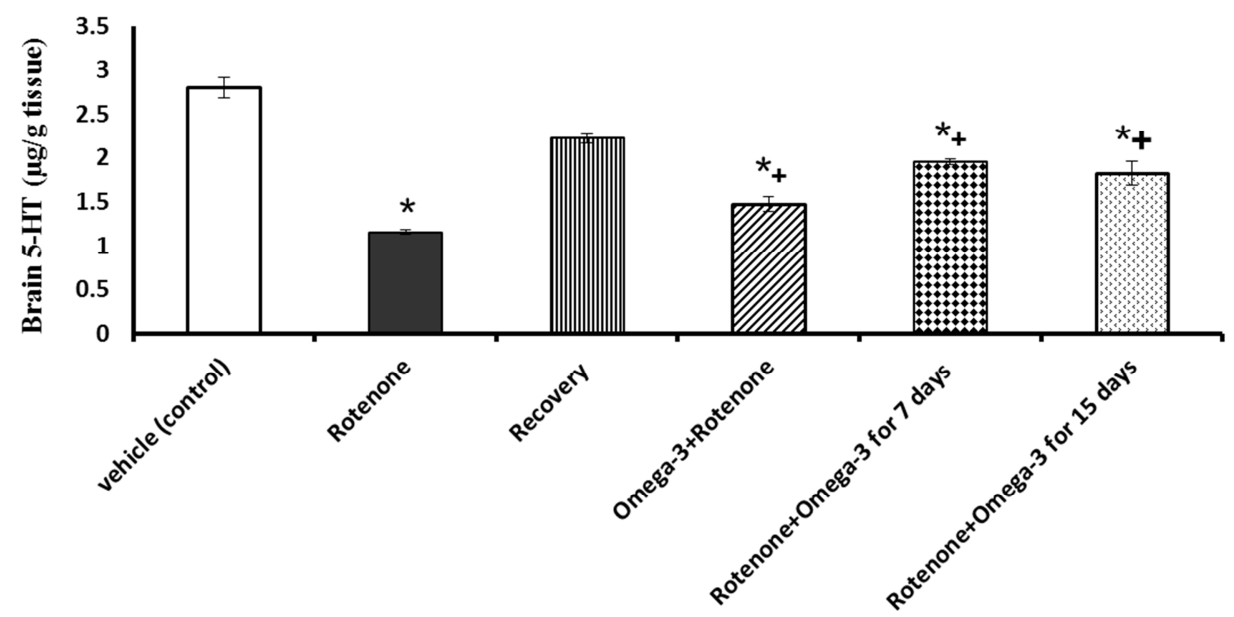

Figure 9. Effects of omega-3 protection or treatment on the level of 5-HT in mice brain of rotenone induced PD model as compared to the vehicle (control)* $P<0.05$ and rotenone ${ }^{+} P<0.05$ groups respectively.

\section{Discussion}

In the present study, a significant increase in the levels of MDA (a marker of lipid peroxidation) and NO together with the significant decrease in reduced glutathione GSH after the daily s. c. injection of rotenone ( $3 \mathrm{mg} / \mathrm{kg}$ every other day) indicate the development of oxidative stress in the brain tissue of rotenone-treated mice.

Rotenone is a potent dopaminergic neurotoxicant owing to its highly lipophilicity, its easy crossing of the blood-brain barrier and its accumulation in subcellular organelles including the mitochondria [37]. It has the ability tobindspecifically to complex I, disrupting mitochondrial respiration and increasing reactive oxygen species (ROS) production [38]. ROS in turn could attack lipid rich membranes of the brain causing an increase in lipid peroxidation [39]. Thus, the present significant increase in the level of lipid peroxidation in the brain tissue could be attributed to the attack of cell membranes of the brain by the free radicals evolved by rotenone.

In addition, the significant increase in the level of NO in the brain tissue recorded in the present study could be mediated by the activation of neuronal nitric oxide synthase (nNOS). Supporting our findings is the study of He et al. (2003) [40] who found that chronic rotenone administration leads to increased activity of NOS in the cerebral tissues. Nitric oxide may inhibit key enzymes of energy metabolism, damage DNA, deplete intracellular glutathione and react with ROS, especially superoxide $\left(\mathrm{O}^{*}\right)$ to form peroxynitrite (ONOO-), a potent oxidant and nitrating agent which has a potent damaging activity [41]. Moreover, the potent oxidant $\mathrm{ONOO}^{-}$may directly oxidize DA [42] causingdopaminergic damage [43]. This could explain the reduced level of dopamine induced by rotenone.

The present recorded reduction of GSH could be attributed to its exhaustion in scavenging the rapidly generating free radicals. Reduced glutathione (GSH) has crucial metabolic importance and its decreased levels might play an important role in inducing oxidative stress in brain [44].
TNF- $\alpha$ a potent pro-inflammatory cytokine has a promoting role in neuroinflammation-mediated progressive degeneration of dopaminergic neurons in PD $[45,46]$. Thus, the increase in brain TNF- $\alpha$ induced by rotenone in the present study could mediate the neuronal inflammation and consequently the dopaminergic damage. Zaitone et al. (2012) [47] observed that rotenone activates the release of TNF- $\alpha$ from microglia.

Accordingly, it could be concluded that the oxidative and nitrosative stress together with the neuroinflammation induced by rotenone in the brain tissue could underlie dopaminergic neurons neurodegeneration and consequently the reduced level of DA.

The present findings indicate that rotenone induced a significant decrease in cerebral AchE activity. According to the DA-Ach balance hypothesis, DA could inhibit acetylcholine releasein the striatum [48]. Therefore, the depletion of dopamine in the striatum could result in dopaminergic/cholinergic imbalance [49] leading toanincrease the level of Ach. This continuous stimulation without inhibition could underlie the characteristic symptoms of tremor, rigidity and muscle fatigue that develop leading to postural instability [50]. AchE is the enzyme responsible for the degradation of acetylcholine. Accordingly, the present decreased activity of AchE could arise from its consumption in the degradation of the abnormal increased Ach content generated during the development of PD.

In agreement with the present findings, Swathi and Rajendra (2014) [51] found that the i. p. injection of rotenone induced a significant decrease in brain DA, NE and 5-HT levels.

The removal of monoamines from the synaptic cleft takes place by a specific vesicular monoamine transporter (VMAT2) [52] which is responsible for repacking monoamine neurotransmitters including DA, NE and 5-HT into their vesicles [53]. This protects them from degradation by monoamine oxidase enzyme (MAO). Watabe and Nakaki (2008) [54] found that rotenone inhibited the activity of VMAT2. In addition, it has been reported that chronic rotenone administration significantly increased the activity of 
MAO enzyme [51]. Therefore the present decrease in the cerebral level of DA, NE and 5-HT may arise from the inhibition of VMAT2 and the increased MAO activity induced by rotenone.

In the present study, the forelimb hanging test was used to assess the disturbance of locomotor activity that represents the main symptom in PD. The present data indicate that the s. c. injection of rotenone (model group) caused a significant decrease in the time of the forelimb hanging test. This finding ensure the establishment of the rat model of PD and reflect a serious model disability. The motor effects are closely linked to the degree of neuronal dysfunction [55]. Therefore, the decrease in DA level may explain the deficit in motor activity induced by rotenone.

The present data revealed that the use of omega-3 either as a protection or a therapy against the mouse model of PD prevented the decrease in GSH level induced by rotenone in the brain tissue, however, it failed to prevent the increased NO level. In addition, only the daily treatment of mouse model of PD with omega-3 for 15 days restored the lipid peroxidation to control-like value.

It has been suggested that DHA, one of omega-3 components, is required for the organization and function of membrane proteins [56]. This may be particularly important in mitochondria, where electron transfer is tightly coupled between the complexes of the embedded electron transport chain. Therefore, accumulation of omega-3 PUFAs within mitochondrial membranes may affect mitochondrial bioenergetics, a suggestion supported by the recent observation that the unsaturation index of mitochondrial membranes in rodents is positively associated with rates of palmitate oxidation [57]. Therefore, omega-3 improves the efficiency of mitochondria either through the greater content of the electron transport complexes, or through the enhanced kinetics of existing proteins [58]. Consequently, omega-3 could prevent the reported inhibition of complex-I induced by rotenone in the mitochondria. This effect may prevent the leakage of single oxygen to the cytoplasm and the formation of free radicals.

In addition, DHA has been found to increase the activities of the antioxidant enzymes glutathione peroxidase and glutathione reductase [59]. Thus, the recorded amelioration in brain GSH levels, in the present study, could be attributed to the stimulatory effect of DHA on the activity of glutathione reductase. This may explain the restoration of GSH level during the protection and therapeutic application.

Production of NO via constitutive nitric oxide synthase has been linked to homeostasis, for instance, the regulation of arterial blood pressure, whereas NO produced after inducible nitric oxide synthase induction appears to be involved in pathophysiological phenomena [60].

The present results recorded a significant increase in NO levels when the mice model of PD was protected or treated with omega-3. In the brain, NO is mainly synthesized in synaptic terminals by a neuronal NOS isoform, acting as a neuromodulator $[61,62]$. In the rat medial preoptic area, NO increased the release of both dopamine and serotonin [63].
Therefore, the present increase in the brain nitric oxide level as a result of omega-3 protection or treatment could play a role in the recovery of dopamine level.

Several studies reported that EPA or fish oil inhibited endotoxin-induced TNF- $\alpha$ production $[64,65]$. In addition, it has been reported that omega-3 PUFAs induce their antiinflammatory effects via reduction of the transcription factor nuclear factor $-\kappa \mathrm{B}$ activation which is a potent inducer of proinflammatory cytokine-like TNF- $\alpha$ [66]. Therefore, the ability of omega-3 to restore the elevated level of TNF- $\alpha$ induced by rotenone could be mediated by inhibiting its production.

Our results showed that both protection and daily treatment (for 7 days) of mouse model of PD with omega-3 reversed the decrease in AchE activity into an increase, however, the enzyme activity returned to control like value after 15 days of daily treatment with omega-3. This may represent the mechanism by which omega-3 acts to attenuate the increase in the cholinergic activity. The control-like value of AchEactivity that has been recorded after 15 days indicates the restoration of cholinergic activity to its normal value. This effect was associated with the recovery in dopamine level and therefore, it may attenuate tremors and dyskinesia associated with PD [67].

Omega-3 PUFAs influence dopaminergic, noradrenergic, serotonergic and GABAergic neurotransmission in particular areas of the brain [68]. Dietary supplementation with omega3 fatty acids increases dopamine levels and D2 receptor binding, and lowers monoamine oxidase B (MAO-B) activity in prefrontal cortex and D2 receptor binding in striatum [69]. Thus, the recovery in dopamine level that was observed after the protection or daily treatment of mice model of PD with omega-3 could be attributed to its inhibitory effect on MAO. This effect could also explain the restoration of NE level.

It is clear from the present results that daily omega-3 therapy for 15 days was effective in preventing the depletion of brain NE level induced in the brain of mouse model of PD, as it restored the changes in NE to control-like value. Unfortunately, the reduced level in cerebral 5HT was still evident when the mice model of PD was protected or treated with omega-3. However, the percentage of decrease was reduced from $-58.7 \%$ in the parkinsonian mice to $-30.2 \%$ and $-34.8 \%$ when the mice model was protected or treated with omega-3 for 7 days and 15 days, respectively. These results indicate a slight improvement that may need more time to be effective.

The present results show that the changes induced by omega-3 in the mice model of PD were associated with noticeable improvement in the motor activity as indicated from the recovered hanging time. This could be attributed to the recovery of DA levels and consequently DA/Ach balance.

\section{Conclusion}

In light of data obtained in the current study. It could be concluded that oral supplementation of omega-3 
polyunsaturated fatty acids (omega-3 PUFAs) may constitute neuroprotective and therapeutic strategies for Parkinson's disease (PD). These beneficial effects of omega-3 could be attributed partially to its antioxidant and anti- inflammatory potential.

\section{References}

[1] de Lau, L. M. L., Breteler, M. M. B. (2006). Epidemiology of Parkinson's disease. Lancet Neurol, 5, 525-535.

[2] Alves, G., Forsaa, E. B., Pedersen, K. F., Gjerstad, M. D., Larsen, J. P. (2008). Epidemiology of Parkinson's disease. J Neurol, 255, 18-32.

[3] de Rijk, M. C., Rocca, W. A., Anderson, D. W., Melcon, M. O., Breteler, M. M., Maraganore, D. M. (1997). A population perspective on diagnostic criteria for Parkinson's disease. Neurology, 48, 1277-1281.

[4] Massano, J., Bhatia, K. P. (2012). Clinical approach to Parkinson's disease: features, diagnosis, and principles of management. Cold Spring Harb Perspect Med, 2, a008870.

[5] Elbaz, A., Moisan, F. (2008). Update in the epidemiology of Parkinson's disease. Curr Opin Neurol, 21, 454-460.

[6] Bronstein, J., Carvey, P., Chen, H., Cory-Slechta, D., DiMonte, D., et al (2009). Meeting report: Consensus statement-Parkinson's disease and the environment: Collaborative on health and the environment and Parkinson's Action Network (CHE PAN) conference, 26-28 June 2007. Environ Health Perspect, 117, 117-121.

[7] Moore, D. J., West, A. B., Dawson, V. L., Dawson, T. M. (2005). Molecular pathophysiology of Parkinson's disease. Annu. Rev. Neurosci., 28, 57- 87.

[8] Smith, M. P., Cass, W. A. (2007). Oxidative stress and dopamine depletion in an intrastriatal 6-hydroxydopamine model of Parkinson's disease. Neuroscience, 144, 1057-1066.

[9] Exner, N., Lutz, A. K., Haass, C., Winklhofer, K. F. (2012). Mitochondrial dysfunction in Parkinson's disease: molecular mechanisms and pathophysiological consequences. EMBO J., 31, 3038-3062.

[10] Giza, E., Gotzamani-Psarrakou, A., Bostantjopoulou, S. (2012). Imaging beyond the striatonigral dopaminergic system in Parkinson's disease. Hell. J. Nucl. Med., 15, 224-232.

[11] Schapira, A. H., Emre, M., Jenner, P., Poewe, W. (2009). Levodopa in the treatment of Parkinson's disease. Eur J Neurol, 16, 982-986.

[12] Pahwa, R., Koller, W. C. (1995). Dopamine agonists in the treatment of Parkinson's disease. Cleve Clin J Med, 62, 212217.

[13] Rivest, J., Barclay, C. L., Suchowersky, O. (1999). COMT inhibitors in Parkinson's disease. Can. J. Neurol. Sci., 26, 3438.

[14] Bassi, S., Albizzati, M. G., Calloni, E., Sbacchi, M., Frattola, L. (1986). Treatment of Parkinson's disease with or phenadrine alone and in combination with L-dopa. Br J ClinPract, 40, 273-275.

[15] Fahn, S. (1996). Is levodopa toxic? Neurology, 47, 184-195.
[16] Idem. (1997). Levodopa-induced neurotoxicity: does it represent a problem for the treatment of Parkinson's disease? CNS Drugs, 8, 376-393.

[17] Barzilai, A., Melamed, E., Shirvan, A. (2001). Is there a rationale for neuroprotection against dopamine toxicity in Parkinson's disease? Cell MolNeurobiol, 21, 215-235.

[18] Hoehn, M. M. M., Elton, R. L. (1985). Low dosages of bromocriptine added to levodopa in Parkinson's disease. Neurology, 35, 199-206.

[19] Su Kp, Huang SY, Chiu TH, Huang KC, Huang CL, Chang HC, Pariante CM. Omega-3 fatty acids for major depressive disorder during pregnancy: results from a randomized, doubleblind, placebo-controlled trial. J Clin Psychiatry 2008; 69, 644-651.

[20] Krauss-Etschmann, S., Shadid, R., Campoy, C., Hoster, E., Demmelmair, H., Jimenez, M., Gil, A., Rivero, M., Veszpremi, B., Decsi, T., Koletzko, B. V., Nutrition and Health lifestyle (NUHEAL) Study Group. (2007). Effects of fish oil and folate supplementation of pregnant women on maternal and fetal plasma concentrations of docosahexaenoic acid and eicosapentaenoic acid: a European randomized multicenter trial. Am J ClinNutr., 85, 1392-400.

[21] Serhan, C. N., Chiang, N., Van Dyke, T. E. (2008). Resolving inflammation: dual anti-inflammatory and pro-resolution lipid mediators. Nat Rev Immunol, 8, 349-361.

[22] Wang, Y., Li, L., Jiang, W., Yang, Z., Zhang, Z. (2006). Synthesis and preliminary antitumor activity evaluation of a DHA and doxorubicin conjugate. Bioorg Med ChemLett., 16, 2974-2977.

[23] Yehuda, S., Rabinovitz, S., Mostofsky, D. I. (1999). Essentialfatty acids are mediators of brain biochemistry and cognitive functions. J. Neurosci. Res., 56, 565-570.

[24] Yurko-Mauro, K., McCarthy, D., Rom, D., Nelson, E. B., Ryan, A. S., Blackwell, A., Salem, N., Jr., Stedman, M., MIDAS Investigators. (2010). Beneficial effects of docosahexaenoic acid on cognition in age-related cognitive decline. Alzheimers Dement., 6, 456-464.

[25] Avramovic, N., Dragutinovic, V., Krstic, D., Colovic, M. B., Trbovic, A., de Luka, S., Milovanovic, I., Popovic, T. (2012). The effects of omega 3 fatty acid supplementation on brain tissue oxidative status in aged wistar rats. Hippokratia., 16, 241-245.

[26] Bousquet, M., Saint-Pierre, M., Julien, C., Salem, N. Jr., Cicchetti, F. (2008). Beneficial effects of dietary omega-3 polyunsaturated fatty acid on toxin-induced neuronal degeneration in an animal model of Parkinson's disease. FASEB J, 22, 1213-1225.

[27] Zimmer, L., Durand, G., Guilloteau, D., Chalon, S. (1999). n3 polyunsaturated fatty acid deficiency and dopamine metabolism in the rat frontal cortex. Lipids, 34, 251.

[28] Vancassel, S., Leman, S., Hanonick, L., Denis, S., Roger, J., Nollet, M., Bodard, S., Kousignian, I., Belzung, C., Chalon, S. (2008). n-3 Polyunsaturated fatty acid supplementation reverses stress-induced modifications on brain monoamine levels in mice. J Lipid Res, 49, 340-348.

[29] Li, K., Huang, T., Zheng, J., Wu, K., Li, D., (2014). Effect of Marine-Derived n-3 Polyunsaturated Fatty Acids on CReactive Protein, Interleukin 6 and Tumor Necrosis Factor $\alpha$ : A Meta-Analysis. PLoS One, 9, e88103. 
[30] Zhang, L., Haraguchi, S., Koda, T., Hashimoto, K., Nakagawara, A. (2010). Muscle atrophy and motor neuron degeneration in human NEDL1 transgenic mice. J. Biomed. Biotechnol., 2011, 1-7.

[31] Ruiz-Larrea, M. B., Leal, A. M., Liza, M., Lacort, M., de Groot, H (1994). Antioxidant effects of estradiol and 2hydroxyestradiol on iron-induced lipid peroxidation of rat liver microsomes. Steroids, 59, 383-388.

[32] Montgomery, H. A. C., Dymock, J. F. (1961). The determination of nitrite in water. Analyst, 86, 414- 416.

[33] Ellman, G. L. (1959). Tissue sulfhydryl groups, Arch Biochem., 82, 70-77.

[34] Ellman, G. L., Courtney, K. D., Andres, V., Featherstone, R. M. (1961). A new and rapid colorimetric determination of acetylcholinesterase activity. Biochem. Pharmacol., 7, 88-95.

[35] Gorun, V., Proinov, I., Baltescu V., Balaban, G., Barzu, O. (1978). Modified Ellman procedure for assay of cholinesterase in crude-enzymatic preparations. Anal. Biochem., 86, 324326.

[36] Ciarlone, A. E. (1978). Further modification of a fluoromertric method for analyzing brain amines. Microchem. J., 23, 9-12.

[37] Talpade, D. J., Greene, J. G. Higgins, D. S. Jr., Greenamyre, J. T. (2000). In vivo labeling of mitochondrial complex I (NADH: ubiquinone oxidoreductase) in rat brain using [(3) H] dihydrorotenone.

[38] Betarbet, R., Sherer, T. B., MacKenzie, G., Garcia-Osuna, M., Panov, A. V., Greenmyre, J. T. (2000). Chronic systemic pesticide exposure reproduces features of Parkinson's disease. Nat. Neurobiol., 3, 1301-1306.

[39] Kale, M., Rathore, N., John, S., Bhatnagar, D. (1999). Lipid peroxidative damage on pyrethroid exposure and alterations in antioxidant status in rat erythrocytes: a possible involvement of reactive oxygen species. Toxicol. Lett., 105, 197-205.

[40] He, Y., Imam, S. Z., Dong, Z., Jankovic, J., Ali, S. F., Appel, S. H., Le, W. (2003). Role of nitric oxide in rotenone-induced nigro-striatal injury. J. Neurochem., 86, 1338-1345.

[41] Korhonen, R., Lahti, A., Kankaanranta, H., Moilanen, E. (2005). Nitric oxide production and signaling in inflammation. Curr. Drug Targets Inflamm. Allergy., 4, 471- 479.

[42] LaVoie, M. J., Hastings, T. G. (1999). Peroxynitrite- and nitrite-induced oxidation of dopamine: implications for nitric oxide in dopaminergic cell loss. J. Neurochem., 73, 25462554 .

[43] Imam, S. Z., Newport, G. D., Itzhak, Y., Cadet, J. L., Islam, F., Slikker, W. Jr., Ali, S. F. (2001). Peroxynitrite plays a role in methamphetamine- induced dopaminergic neurotoxicity: evidence from mice lacking neuronal nitric oxide synthase gene or overexpressing copper-zinc superoxide dismutase. J. Neurochem., 76, 745-749.

[44] Dickinson, D. A., Forman, H. J. (2002). Cellular glutathione and thiols metabolism. Biochem. Pharm., 64, 1019-1026.

[45] Frankola, K. A., Greig, N. H., Luo, W., Tweedie, D. (2011). Targeting TNF-alpha to elucidate and ameliorate neuroinflammation in neurodegenerative diseases. CNS and Neurological Disorders - DrugTargets, 10, 391-403.

[46] Montgomery, S. L., Bowers, W. J. (2012). Tumor necrosis factor alpha and the roles it plays in homeostatic and degenerative processes within the central nervous system. Jounal of Neuroimmune Pharmacology, 7, 42-59.

[47] Zaitone, S. A., Abo-Elmatty, D. M., Elshazly, S. M. (2012). Piracetam and vinpocetine ameliorater otenone-induced Parkinsonism in rats. Indian J. Pharmacol., 44, 774-779.

[48] Stoof, J. C., Drukarch, B., de Boer, P., Westerink, B. H., Groenewegen, H. J. (1992). Regulation of the activity of striatal cholinergic neurons by dopamine. Neuroscience, 47, 755-770.

[49] Aosaki, T., Miura, M., Suzuki, T., Nishimura, K., Masuda, M. (2010). Acetylcholine-dopamine balance hypothesis in the striatum. GeriatrGerontolInt, 10, 148-157.

[50] Swathi, G., Bhuvaneswar, C., Rajendra, W. (2013). Alterations of cholinergic neurotransmission in rotenone induced parkinson's disease: protective role of bacopamonnieri. Int. J. Pharm. Biol. Sci., 3, 286-292.

[51] Swathi, G., Rajendra, W. (2014). Protective role of bacopamonnierion induced Parkinson's disease with particular reference to catecholamine system. Int. J. Pharm. Pharm. Sci., $6,379-382$.

[52] Njus, D., Kelley, P. M., Hardabek, G. J. (1986). Bioenergetics of secretory vesicles. Biochim. Biophys. Acta., 853, 237-265.

[53] Nirenberg, M. J., Chan, J., Liu, Y., Edwards, R. H., Pickel, V. M. (1996). Ultrastructural localization of the vesicular monoamine transporter-2 in midbrain dopaminergic neurons: potential sites for somatodendritic storage and release of dopamine. J. Neurosci., 16, 4135- 4145.

[54] Watabe, M., Nakaki, T. (2008). Mitochondrial complex I inhibitor rotenone inhibits and redistributes vesicular monoamine transporter 2 via nitration in human dopaminergic SH-SY5Y cells. Mol. Pharmacol., 74, 933-940.

[55] Schwarting, R. K., Bonatz, A. E., Carey, R. J., Huston, J. P. (1991). Relationships between indices of behavioral asymmetries and neurochemical changes following mesencephalic 6-hydroxydopamine injections. Brain Res., $554,46-55$.

[56] Infante, J. P., Huszagh, V. A. (2000). Secondary carnitine deficiency and impaired docosahexaenoic (22: 6n-3) acid synthesis: a common denominator in the pathophysiology of diseases of oxidative phosphorylation and $\beta$-oxidation. FEBS Lett, 468, 1-5.

[57] Holloway, G. P., Fajardo, V. A., McMeekin, L., LeBlanc, P. J. (2012). Unsaturation of mitochondrial membrane lipids is related to palmitate oxidation in subsarcolemmal and intermyofibrillar mitochondria. J Membr Biol., 245, 165-176.

[58] Peoples, G. E., McLennan, P. L. (2010). Dietary fish oil reduces skeletal muscle oxygen consumption, provides fatigue resistance and improves contractile recovery in the rat in vivo hindlimb. Br J Nutr, 104, 1771-1779.

[59] Wang, J. Y., Sekine, S., Saito, M. (2003). Effect of docosahexaenoic acid and ascorbate on peroxidation of retinal membranes of ODS rats. Free Radic. Res., 37, 419-424.

[60] Moncada, S., Palmer, R. M. J., Higgs, E. A. (1991). Nitric oxide: physiology, pathophysiology, and pharmacology. J. Pharm. Exp. Ther., 43, 109-141. 
[61] Garthwaite, J. (2008). Concepts of neural nitric oxidemediated transmission. European Journal of Neuroscience, 27, 2783-2802.

[62] Steinert, J. R., Chernova, T., Forsythe, I. D. (2010). Nitric oxide signaling in brain function, dysfunction, and dementia. Neuroscientist, 16, 435-452.

[63] Lorrain, D. S., Hull, E. M. (1993). Nitric oxide increases dopamine and serotonin release in the medial preoptic area. Neuroreport, 5, 87-89.

[64] Lo, C. J., Chiu, K. C., Fu, M., Lo, R., Helton, S. (1999). Fish oil decreases macrophage tumor necrosis factor gene transcription by altering the NF kappa B activity. J. Surg. Res., 82, 216-221.

[65] Babcock, T. A., Novak, T., Ong, E., Jho, D. H., Helton, W. S., Espat, N. J. (2002). Modulation of lipopolysaccharidestimulated macrophage tumor necrosis factor- $\alpha$ production by $\omega-3$ fatty acid is associated withdifferential cyclooxygenase- 2 protein expression and is independent of interleukin-10. J. Surg. Res., 107, 135-139.

[66] Siriwardhana, N., Kalupahana, N. S., Moustaid-Moussa, N. (2012). Health benefits of n-3 polyunsaturated fatty acids: eicosapentaenoic acid and docosahexaenoic acid. Adv Food Nutr Res., 65, 211-222.

[67] Won, L., Ding, Y., Singh, P., Kang, U. J. (2014). Striatal cholinergic cell ablation attenuates L-DOPAinduceddyskinesia in Parkinsonian mice. J. Neurosci., 34, 3090-3094.

[68] Das, U. N., Fams. (2003). Long-chain polyunsaturated fatty acids in the growth and development of the brain and memory. Nutrition, 19, 62-65.

[69] Chalon, S., Delion-Vancassel, S., Belzung, C., Guilloteau, D., Leguisquet, A., Besnard, J. C., Durand, G. (1998). Dietary fish oil affects mono aminergicneuro transmission and behavior in rats. J Nutr., 128, 2512-2519. 